\title{
Orthopaedic training during COVID-19 pandemic: should action be taken?
}

\author{
Ahmed Hazem Abdelazeem ${ }^{1} \cdot$ Ahmed Mohamed Khedr $^{1} \cdot$ Marius M. Scarlat $^{2}$ \\ Published online: 14 January 2022 \\ (c) The Author(s) under exclusive licence to SICOT aisbl 2022
}

Since COVID-19 was declared a pandemic in March 2020, drastic changes have affected all aspects of life [1]. Education was not far apart from the dynamic situation, particularly orthopaedic residency, and fellowship training. While medical specialties were the workhorse of the medical service, orthopaedic surgery and other surgical subspecialties were pushed aside. Twenty-five to fifty percent of orthopaedic trainees were redeployed to medical and ICU services and recruited as frontline responders [2, 3]. For those who remained in orthopaedic services, the workload was reduced, or trainees worked in alternate teams with a specific period on-duty followed by a certain period in isolation or off-duty $[3,4]$. The operating time spent by trainees for trauma and elective surgeries and the clinic was significantly reduced [5, 6]. In the UK, the authorities urged the consultants to be the first surgeons in surgeries that otherwise a trainee can do to decrease the operative time [7]. The pandemic even changed the practice in some regions of the world where trauma cases that were typically managed surgically were treated conservatively [8]. Although the pattern of loss of training opportunities was not uniform globally [3], we assume that all world regions were affected to some extent.

To provide an updated evaluation of the effect of the COVID-19 pandemic on orthopaedic training worldwide, we conducted a concise 6-questions survey. It involved a single choice answer to questions including nationality, professionalism, personal opinion of the level of affection, any actions, or modifications from the notational body responsible for training, and finally, subjective opinion about the negative drawback on developing competent future consultants. The survey was accomplished under the umbrella

Marius M. Scarlat

mscarlat@gmail.com

1 Department of Orthopaedics and Traumatology, Kasr-Alainy Hospital, Cairo University, Cairo, Egypt

2 Groupe ELSAN, Clinique Chirurgicale St Michel, Toulon, France of the International Society of Orthopaedics Surgery and Traumatology (SICOT) and sent to orthopaedic surgeons worldwide in the email database of the headquarter, mostly to the SICOT members.

We received 240 responses from the 22 nd of November till the 6th of December 2021. Ten responses were excluded because of repetition or inconsistent answers. Finally, answers from 230 orthopaedic surgeons from 76 different nations representing the six continents were included and analyzed (Fig. 1). We categorized the respondents according to professionalism into four groups; surgeons involved in training supervision, either a university staff member or staff in an educational hospital, surgeons not involved in any training program, and trainees. Seventy percent of the replies were from surgeons involved in different training programs supervision worldwide (Fig. 2).

Seventy-eight percent of the respondents agreed that the COVID-19 pandemic had a moderate to high effect on the current training programs (Fig. 3a). A funnel-shaped diagram analysis of different groups showed similar views distribution with the majority voting for the moderate, followed by high affection. Trainees were the most pessimistic, with none voting for the "very low" affection, unlike the other three groups (Fig. 3b). Fifty-nine percent reported no action from their national training body to counteract the situation (Fig. 4). Few reported extensions of the training program period, while many mentioned shifting to digitalization and telecommunication for education. Almost half of the respondents were pessimistic and agreed that this situation would affect the development of competent orthopaedic surgeons in the future. Only $19 \%$ think that trainees will be capable of catching up and upgrading their knowledge (Fig. 5a). Again, the funnel-shaped diagram showed a comparable sights distribution between the different groups, revealing a global agreement and sending a clear message about frightening worries from the effect of this pandemic on the future orthopaedic surgeons' capability and competency (Fig. 5b). 


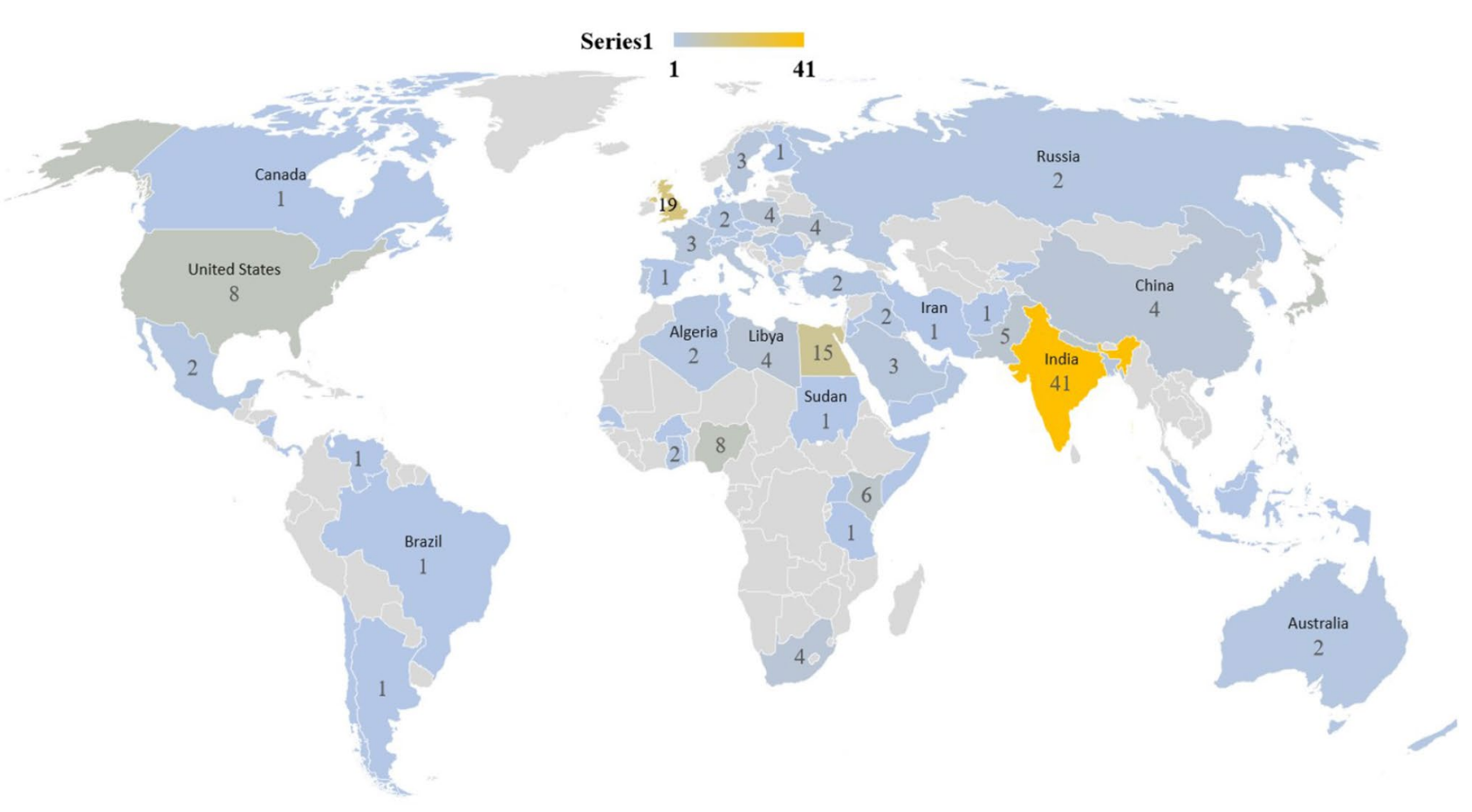

Fig. 1 Respondents' nationalities and distribution numbers

Fig. 2 Respondents' profession four categories distribution

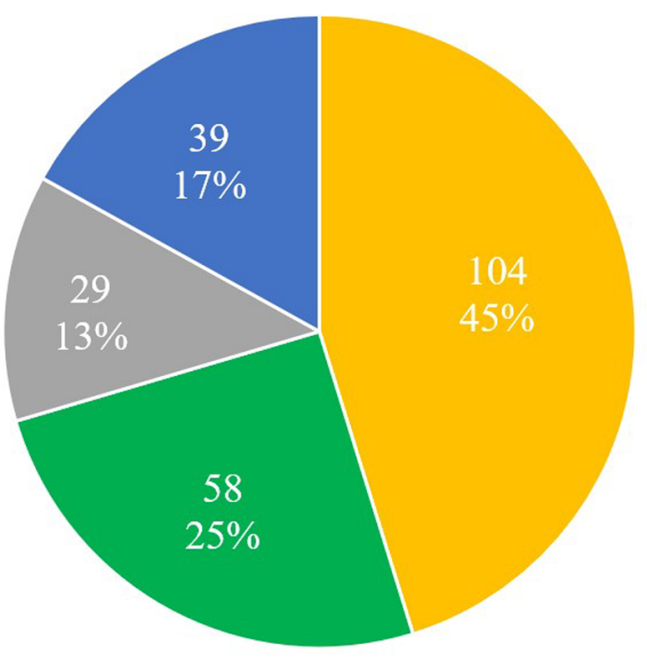

- Consultant in an educational hospital

- University staff member

- Trainee
- Surgeon; not involved in any training program
The results of our recent survey were comparable with previously published data. Seventy-eight percent of our respondents believed that the pandemic harmed training. Bodansky et al. reported a $43 \%$ decrease in training opportunities in the UK due to the pandemic [7]. Kumar et al. noted an $86 \%$ reduction in surgical exposure for residents in India [9]. An et al. described the variable magnitude of disruption of the training program in the USA according to the geographical distribution of the residency program [4]. In a global cross-section analysis, Bosco et al. reported a 24.7-h reduction in the time spent for training in orthopaedic residency and $50.2 \%$ of residents performed tasks not related to their residency training during the first wave [3]. Faria et al. surveyed the redeployed orthopaedic trainees if 
a

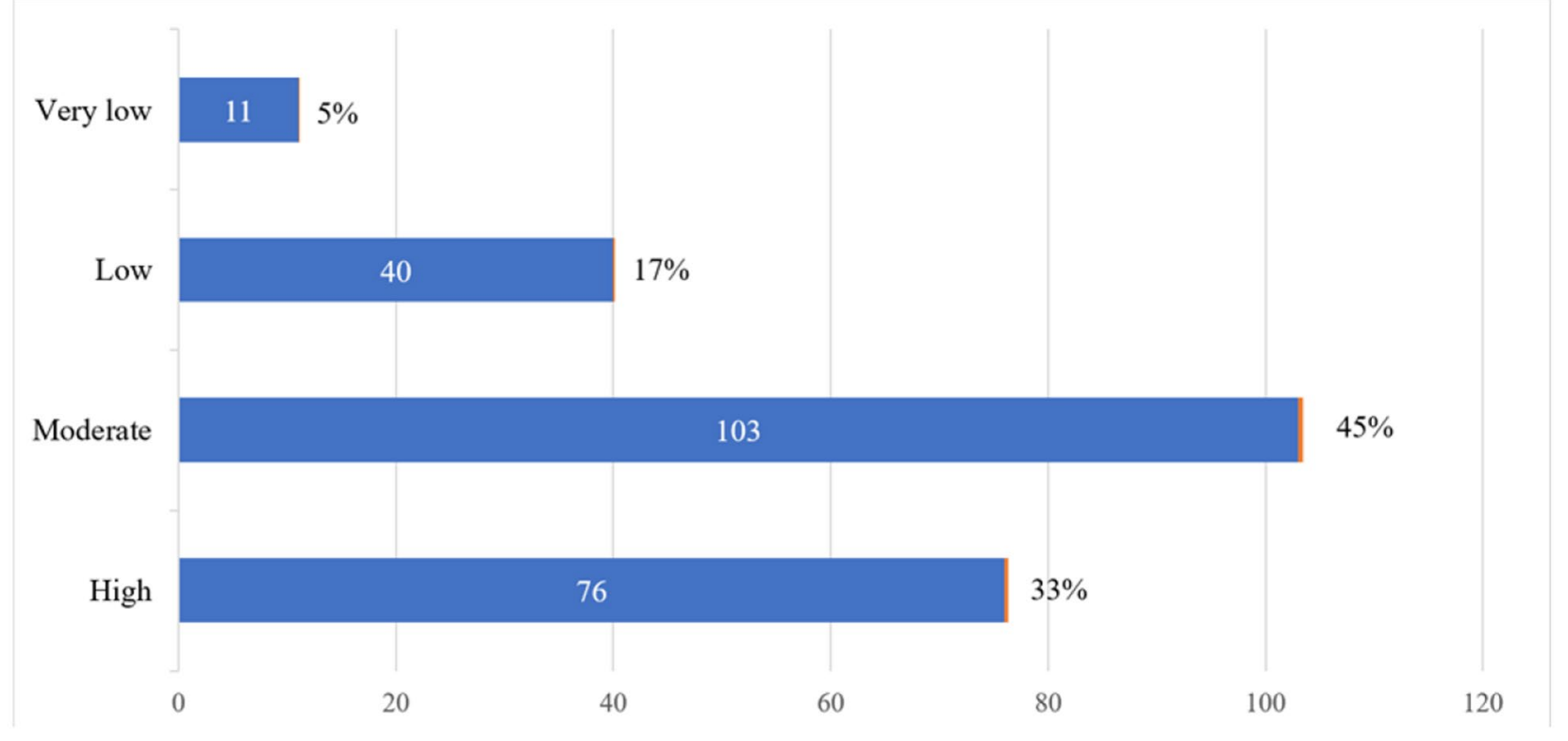

b

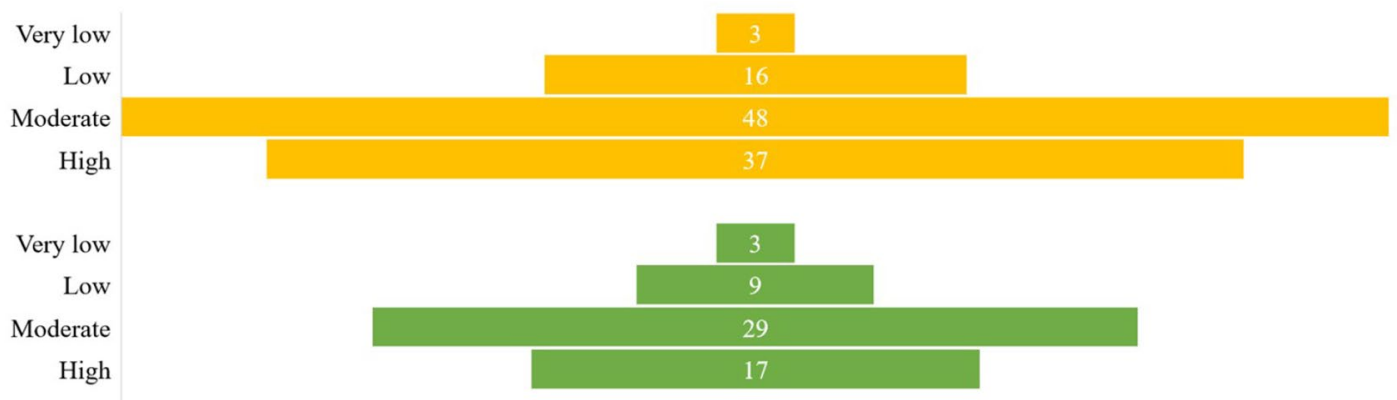

Very low

Low

Moderate

High

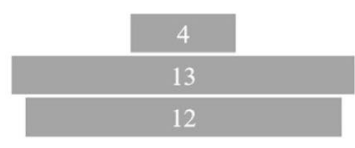

Very low

Low

Moderate

High

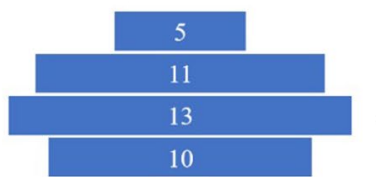

Consultant in an educational hospital

University staff member

Trainee

Surgeon; not involved in any training program

Fig. 3 a Personal opinion of the level of affection of the COVID-19 pandemic on orthopaedic training. b Personal opinion of the level of affection of the COVID-19 pandemic on orthopaedic training according to different professions views

they found the skills, they gained from working in other subspecialties would benefit them in their orthopaedic careers. However, most responded that these skills would not be applicable in their careers [10]. Similarly, Gonzi et al. measured whether trainees felt that deployment to the emergency department added to their training on a Likert scale from 1 (disagree) to 5 (agree). The mean response was 1.33 [2]. Another variable was the training service where the trainee was enrolled. When spine fellows and sports medicine fellows were surveyed for the effect of the pandemic, $62.2 \%$ of spine fellows compared to $80.4 \%$ of sports fellows reported $>50 \%$ reduction in the number of cases they operated upon due to cancellation of elective surgery [11, 12]. A study from Ireland indicated that elective surgery was significantly reduced during the lockdown. However, trauma surgery did not decrease significantly. Interestingly, they reported an increase in hemiarthroplasties compared to the same time frame in 2018 and 2019 [13]. 


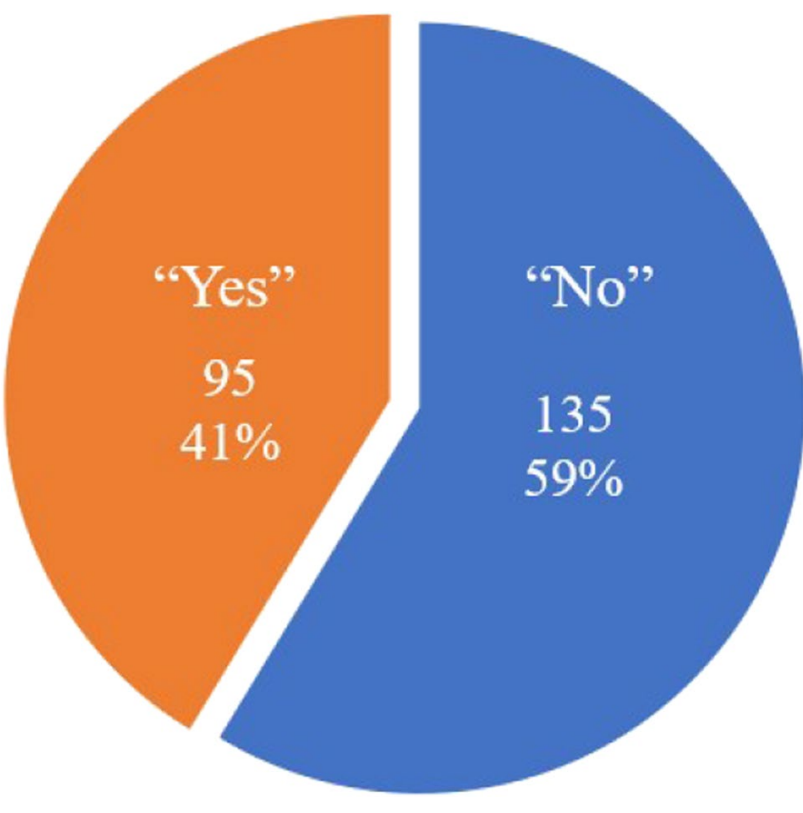

Fig. 4 The response of the national training body to COVID pandemic to cope with the defects and maintain high standards of training

We cannot ignore the negative psychological impact of the COVID-19 pandemic that casted its shadow on the bumpy trainees' road. In our study, the trainee group answer revealed this; none of them voted for the "very low" effect on the training programs as the other groups did. Similarly, only two voted for no effect when assessing the future competency. This reflects the concerns of trainees regarding the resumption of their regular training, the schedules of their exit exams and their ability to catch up for the lost educational opportunities. Moreover, different studies reported a significant decrease in the quality of life (QOL) among trainees with increased anxiety levels [3, 5, 12]. The most common stressors were transmitting COVID-19 infection to the family due to hospital exposure and failure to fulfill training requirements due to elective surgery and clinics suspension $[3,5]$.

Despite the pandemic's negative effect on the training in general, Barlow et al. in a study from Poland highlighted some positive impacts. The presence of a consultant in the trauma operation room, which was not the rule before the pandemic, led to an increase in the proportion of satisfactory radiographic position of the lag screw and tip-apex distance (TAD) $<20 \mathrm{~mm}$ during dynamic hip screw fixation of hip fractures from 29.6 to $42.3 \%$ [14]. Another positive impact was the exposure of this cohort of trainees to video consultations and their training for virtual clinic models [7].

An integral part of the training program is the trainee education through the didactics and lectures. Face-to-face lectures and meetings have been suspended since the beginning of the pandemic worldwide. The good thing is that these specific educational activities continued through different virtual platforms [3, 4]. However, virtual education methods' satisfaction was perceived as less than the traditional methods $[3,5]$.

Another issue that we would like to highlight is how to define the negative effect on different training aspects. While some effects can be measured by comparing the number of cases operated upon and clinic times that trainees log in, other aspects could be difficult to highlight, being non-measurable. These include the development of communication skills with the hospital staff and the patients, the ability to communicate with patients about adverse events, research skills, and others. The training systems routinely provided these skills before the pandemic, which is very important in building up the orthopaedic surgeon's competent personality besides the surgical skills and knowledge.

Surprisingly, reactions from the responsible bodies for training assessment and validation were limited and sporadic. Only $41 \%$ of our surveyed population stated that their national body took action. The description of these actions was very variable and inconsistent, reflecting a lack of understanding or underestimating the situation. Minor clear reactions were reported from some countries. In the USA, the Accreditation Council for General Medical Education (ACGME) would accept a 10\% decrease in total cases and time requirements in 2019 and 2020. In addition, the ACGME will rely on the assessment of the program director of the competence of the trainees [15]. In the UK, two outcomes were added to the Annual Review of Competence Progression (ARCP), 10.1 and 10.2. Both reflect the progress during the COVID-19 period, with outcome 10.1 denoting optional additional training time and 10.2 indicating that extra training time is required [16]. In addition, exit exams were postponed and rescheduled in different areas in the world [17]. Other authors suggested using virtual exams models [18]. A global cross-sectional survey showed that $70.6 \%$ of participants had their exit exams rescheduled, and 49.5\% had their residencies extended [3].

The final question is always how this will affect the development of a competent orthopaedist in the future. Answer from our survey revealed global doubtfulness, with $47 \%$ confidently stating yes and $34 \%$ suspicious (Fig. 5a). A survey for spine fellows in North America showed that $95.5 \%$ of the fellows believed that COVID-19 would not affect their ability to complete their training. However, $32 \%$ of the program directors heard concerns from their fellows about being ready for their practice. Twenty-five percent of the spine fellows were concerned about job opportunities [12]. A similar survey of sports medicine fellows yielded similar responses [11]. A survey from Ireland showed that $35 \%$ of trainees felt that their training should be extended to fulfill the training requirements [13]. All these reflects a global uncertainty in 
a

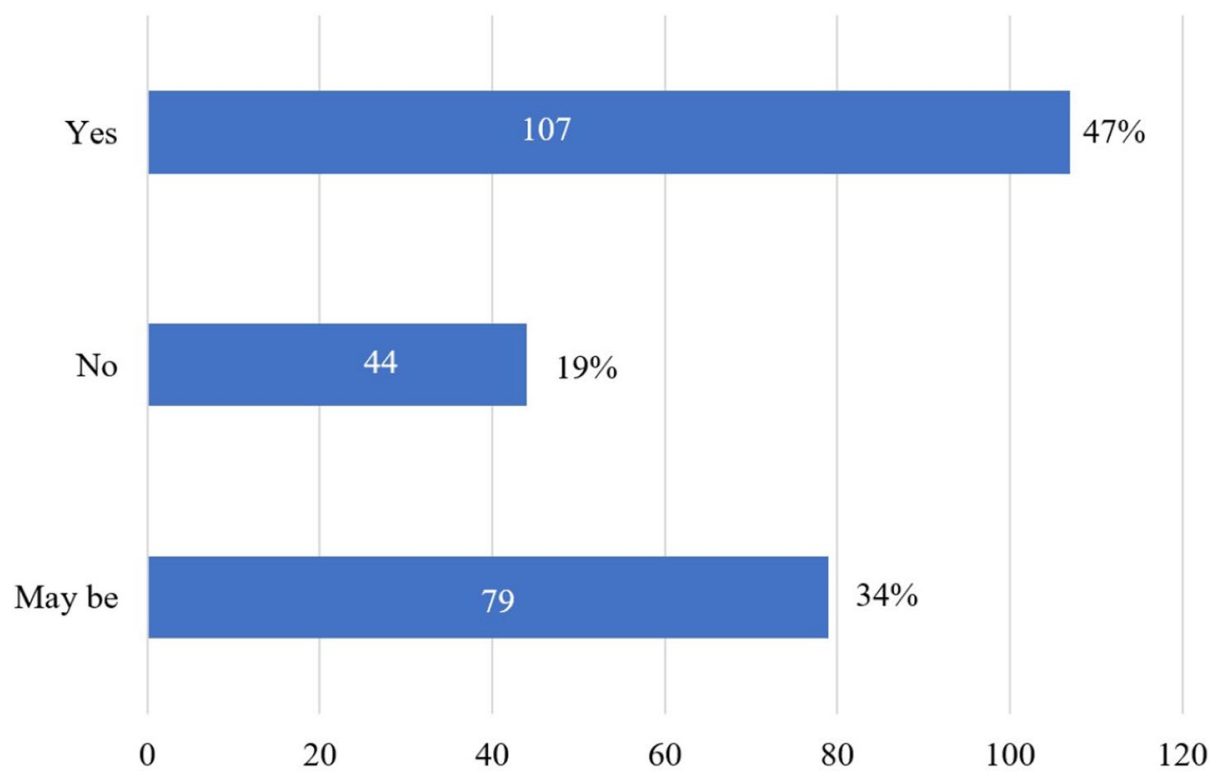

b

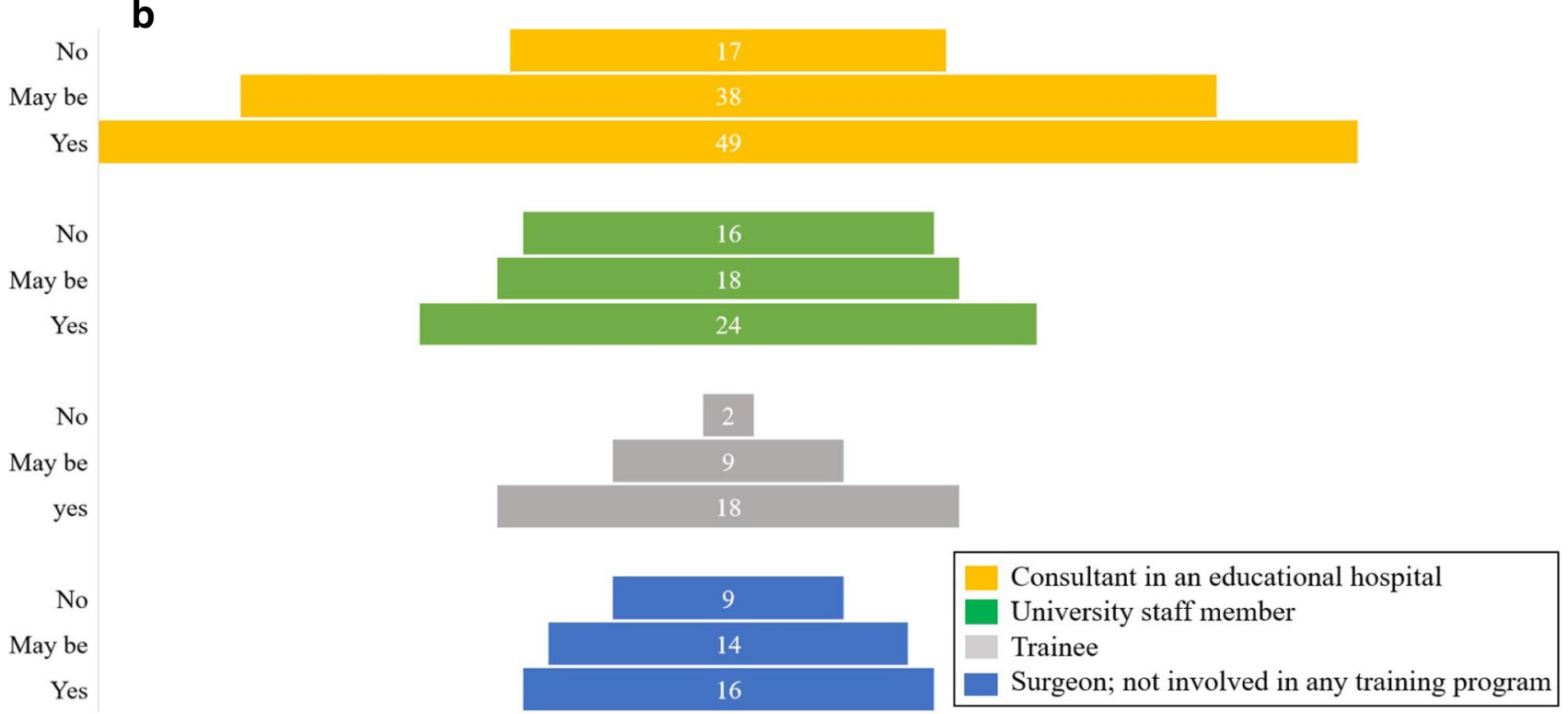

Fig. 5 a The future expectation of the effect of the COVID pandemic on the development of a competent orthopaedic consultant. b The future expectation of the effect of the COVID pandemic on the devel- opment of a competent orthopaedic consultant according to different profession views

Another added value is having $80 \%$ of these surgeons deal with training care either in university or educational hospitals worldwide.

We believe that the unfortunate trainees who had their training during the COVID era should not pay for the lower educational opportunities. However, the training bodies worldwide should ensure that the training systems are still producing competent and safe orthopaedic surgeons by greater involvement of the training hospitals, program directors and faculty in the decision of graduation or extending in the literature, which collected responses from trainees. 
the education to catch up with the lost training disciplines. This can be done either in a case-by-case assessment or by assessing the areas which suffered from heavier infection rates and more periods of training disruption.

\section{References}

1. WHO Director-General's opening remarks at the media briefing on COVID-19 - 11 March 2020. https://www.who.int/dg/speec hes/detail/who-director-general-s-opening-remarks-at-the-mediabriefing-on-covid-19---11-march-2020. Accessed 26 May 2020

2. Gonzi G, Gwyn R, Rooney K et al (2020) The role of orthopaedic trainees during the COVID-19 pandemic and impact on postgraduate orthopaedic education. Bone Jt Open 1:676-682. https:// doi.org/10.1302/2633-1462.111.bjo-2020-0095.r1

3. Bosco A, Tay HW, Aleem I et al (2021) Challenges to the orthopedic resident workforce during the first wave of COVID-19 pandemic: lessons learnt from a global cross-sectional survey. $\mathrm{J}$ Orthop 27:103-113. https://doi.org/10.1016/J.JOR.2021.09.001

4. An TW, Henry JK, Igboechi O et al (2020) How are orthopaedic surgery residencies responding to the COVID-19 pandemic? An Assessment of Resident Experiences in Cities of Major Virus Outbreak. J Am Acad Orthop Surg 28:E679-E685. https://doi.org/10. 5435/JAAOS-D-20-00397

5. Chang DG, Park JB, Baek GH et al (2020) The impact of COVID19 pandemic on orthopaedic resident education: a nationwide survey study in South Korea. Int Orthop 44:2203-2210. https://doi. org/10.1007/s00264-020-04714-7

6. Jayatilaka MLT, As-Sultany M, Gabr A et al (2021) Collaborative Overview of coronaVIrus impact on ORTHopaedic training in the UK (COVI - ORTH UK). Surgeon 19:e331-e337. https://doi.org/ 10.1016/j.surge.2021.02.007

7. Bodansky D, Thornton L, Sargazi N et al (2021) Impact of COVID-19 on UK orthopaedic training. Bull R Coll Surg Engl 103:38-42. https://doi.org/10.1308/rcsbull.2021.11

8. BOAST - Management of patients with urgent orthopaedic conditions and trauma during the coronavirus pandemic (Archived). https://www.boa.ac.uk/resources/boast-management-of-patientswith-urgent-orthopaedic-conditions-and-trauma-during-the-coron avirus-pandemic.html. Accessed 28 Dec 2021
9. Kumar GSP, Yadav AK, Harsoor A et al (2021) Impact of COVID19 on orthopaedic residents-an Indian perspective. OTA Int Open Access J Orthop Trauma 4:e096. https://doi.org/10.1097/ oi9.0000000000000096

10. Faria G, Tadros BJ, Holmes N et al (2020) Redeployment of the trainee orthopaedic surgeon during COVID-19: a fish out of water? Acta Orthop 91:650-653. https://doi.org/10.1080/17453 674.2020.1824155

11. Swiatek PR, Weiner J, Alvandi BA et al (2021) Evaluating the early impact of the COVID-19 pandemic on sports surgery fellowship education. Cureus 13. https://doi.org/10.7759/cureus.12943

12. Swiatek PR, Weiner JA, Butler BA et al (2021) Assessing the early impact of the COVID-19 pandemic on spine surgery fellowship education. Clin Spine Surg 34:E186-E193. https://doi.org/ 10.1097/BSD.0000000000001073

13. Sheridan GA, Hughes AJ, Quinlan JF et al (2020) Quantifying the impact of the COVID-19 pandemic on orthopaedic trainees. Bone Jt Open 1:645-652. https://doi.org/10.1302/2633-1462.110. bjo-2020-0149.r1

14. Barlow E, Larsen MP, Vijayasurej K et al (2021) The impact of COVID-19 on dynamic hip screw fixation and training. Pol Merkur Lekarski 49:273-277

15. ABMS, ACGME (2020) ABMS and ACGME joint principles: physician training during the COVID-2019 pandemic. https:// www.abms.org/news-events/abms-and-acgme-joint-principlesphysician-training-during-the-covid-2019-pandemic/. Accessed 16 Dec 2021

16. JCST Contingency Plan for ARCP 2020. https://www.asit.org/ news/jcst-contingency-plan-for-annual-review-of-competenceprogression-arcp--2020/nwc11199. Accessed 19 Dec 2021

17. James HK, Pattison GTR (2021) Disruption to surgical training during Covid-19 in the United States, United Kingdom, Canada, and Australasia: a rapid review of impact and mitigation efforts. J Surg Educ 78:308-314. https://doi.org/10.1016/j.jsurg.2020.06. 020

18. Iyengar KP, Jain VK, Vaishya R (2021) Virtual postgraduate orthopaedic practical examination: A pilot model. Postgrad Med J 97:650-654

Publisher's note Springer Nature remains neutral with regard to jurisdictional claims in published maps and institutional affiliations. 